\title{
Docencia innovadora en cirugía
}

\author{
Boris Marinkovic G. ${ }^{1}$
}

\section{Innovative teaching in surgery}

Innovative education in surgery is frequently associated with incorporating modern technology to facilitate learning in our specialty. Nevertheless, innovation in education is more dependent on restructuring the educational processes than introducing technology alone. This is underpinned by a consistent amount of evidence generated by education professionals, that usually conduct research under the paradigm of social sciences, which differ widely from the ways in which surgeons understand the world. However, during the last 40 years, there has been a well-recognized shift in the way we surgeons educate. It has been claimed that the determinants of such changes have been the introduction of minimal invasive procedures, sociocultural changes and the development of modern education theories. Our surgical scientific society still have the challenge of promoting the training of surgical educators, stablishing national standards according to the experience of the developed world and regulating undergraduate and graduate curricula by introducing modern educational concepts like competency-based education and professionalism. Our major goal is to professionalize medical education in our country, focusing in the learning process of the residents or students. This imply among other aspects, to change the focus from educating on a rigid set of technical procedures to train professionals that are capable of responding competently to a world that is in a rapid and constant change. I believe that this is the only way to innovate in surgical education. Once we have reach this, then we will be able to focus on the modern technology that can help us achieve such goals.

Key words: surgery; training; teaching; innovation.

\section{Resumen}

La docencia innovadora en cirugía la asociamos habitualmente al uso de tecnología moderna para mejorar la educación que realizamos en nuestra especialidad. Sin embargo, en educación, la innovación está relacionada con la incorporación de aspectos más teóricos que tecnológicos. Esto está respaldado por una creciente cantidad de evidencia conducida principalmente por educadores, quienes utilizan la mirada de las ciencias sociales, que difieren ampliamente de cómo validamos la evidencia los cirujanos. Pese a esto, en los últimos 40 años, hemos visto globalmente como ha habido un importante cambio paradigmático en el modelo educativo con el que educamos en cirugía. Este ha sido impulsado básicamente por la introducción de técnicas mínimamente invasivas, cambios sociales y el progreso de las ciencias de la educación. Como sociedad científica tenemos aun el desafío de impulsar la formación de educadores quirúrgicos, establecer estándares nacionales según la experiencia de países líderes en la materia y regular los currículos de pre y postgrado introduciendo conceptos educacionales modernos como el profesionalismo y la educación basada en competencias. Nuestra meta debería ser una sola: profesionalizar la educación quirúrgica en nuestro país poniendo el foco en el residente o en el estudiante. Esto implica entre otras cosas, que se cambie el objetivo de formar profesionales que dominen una batería rígida de procedimientos a prepararlos para enfrentar competentemente un mundo profesional que se encuentra en constante cambio y evolución. Sólo así estaremos realmente realizando innovación en docencia quirúrgica y podremos enfocarnos entonces en la tecnología.

Palabras clave: cirugía; entrenamiento; docencia; innovación.
Departamento de Cirugía Oriente, Universidad de Chile. Santiago, Chile.

Recibido el 28 de marzo de 2020 y aceptado para publicación el 10 de abril de 2020

Correspondencia a:

Dr. Boris Marinkovic G. borismarinkovic@gmail.com 


\section{Introducción}

Es muy probable que, si nos disponemos a leer un trabajo sobre Docencia Innovadora en Cirugía, esperemos encontrar información sobre cómo usar elementos tecnológicos modernos para mejorar la enseñanza de nuestra especialidad. Esta asociación, está dada probablemente por el hecho de que la innovación en la práctica misma de la disciplina, está definida por la introducción de técnicas o instrumentos que utilizan tecnología avanzada para mejorar la seguridad de los procedimientos y minimizar su invasividad. Sin embargo, en el campo de la educación, las innovaciones más radicales y transformativas se fundamentan en aspectos más conceptuales que tecnológicos.

La definición académica de innovación en educación superior es "un producto, proceso, método organizacional u organización en sí en el que se mejora significativamente el impacto en las actividades de educación superior"'. De esta forma, la mejor definición de docencia innovadora en cirugía es aquella que es capaz de incorporar prácticas novedosas, pero necesariamente orientadas a impactar en forma positiva en cómo aprenden la especialidad los estudiantes o residentes.

Es importante destacar que la educación quirúrgica en sí, es un concepto ya innovador, puesto que corresponde a un área emergente de la academia. $\mathrm{Si}$ se busca literatura sobre educación quirúrgica, más del $50 \%$ de los artículos al respecto se han generado tan sólo en los últimos 5 años ${ }^{2}$. Esto ocurre también con la formación en docencia quirúrgica donde a la fecha sólo existen dos programas de magister en el mundo y desde hace no más de 15 años ${ }^{3,4}$.

Para entender el concepto de innovación en docencia quirúrgica, es imprescindible considerar la historia de ésta en el mundo y cómo ha evolucionado hasta la actualidad. Asimismo, es necesario hacer un análisis del contexto sociopolítico mundial y local para analizar cuál es la situación de la educación quirúrgica en nuestro país. Esto nos permitirá evaluar cuáles son los desafíos que tenemos como sociedad científica.

\section{Desarrollo histórico de la educación en cirugía}

Hasta 1980 la forma como se educaba en cirugía se había mantenido más o menos estable y herméti$\mathrm{ca}^{5}$. El modelo norteamericano basado en el modelo "maestro-aprendiz" introducido por William Halsted en 1889 , se había impuesto en el mundo como la forma predominante de docencia en el postgrado ${ }^{6}$. Bajo este marco, la exposición prolongada al ejercicio clínico supervisado de la cirugía es el mecanismo principal que asegura la competencia quirúrgica al final del tiempo estipulado para ello. El residente adquiere así una responsabilidad creciente a medida que aumenta la confianza de sus mentores y disminuye el nivel de su supervisión. El cirujano formado repite esta forma una vez más con sus discípulos y se perpetúa así el modelo educativo ${ }^{7}$. La evaluación y el progreso dentro de este proceso no está marcado por indicadores sistemáticos que dependan del diseño instruccional de los programas, sino más bien en opiniones de los miembros con más experiencia sin que haya mucha formalidad ni control ${ }^{5}$.

Desde esa fecha en adelante todo empezó a cambiar. Hay cierto consenso en que el cambio se inició con la disrupción que produjo la tecnología. La introducción de la cirugía mínimamente invasiva vino a cuestionar el modelo educativo ya que se empezaron a requerir en forma súbita un conjunto de habilidades técnicas nuevas y distintas a las ya utilizadas en cirugía abierta de cavidades ${ }^{6}$. No sólo los cirujanos en formación debían aprenderlas sino también sus maestros. La larga exposición a la cirugía abierta no resultaba suficiente para asegurar la habilidad en la ejecución de la cirugía mínimamente invasiva y, por lo tanto, no quedaba otra alternativa que recurrir a métodos educacionales estructurados y distintos a los existentes ${ }^{5}$. Los cirujanos nos vimos por primera vez en la necesidad de innovar en docencia quirúrgica.

A esta avalancha tecnológica, se sumó una serie de cambios socioculturales, sobre todo en países anglosajones, junto con un cuestionamiento general a las estructuras de autoridad que hasta ese entonces se mantenían estables 5 . La publicación en 1999 del libro "Errar es humano: Construyendo un sistema de salud seguro" hizo evidente que hasta unas 98.000 personas mueren al año debido a errores médicos en EE.UU., muchos de los cuales ocurrían justamente en procedimientos quirúrgicos ${ }^{8}$. Además, se produjeron casos emblemáticos de situaciones poco éticas como el escándalo de los cirujanos cardíacos de Bristol, donde se descubrió que algunos de sus miembros continuaban operando niños pequeños aún cuando el equipo completo estaba en conocimiento de que tenían una clara mayor mortalidad que sus colegas?.

El escenario se hizo más complejo por la judicialización de la medicina, envejecimiento de la población ${ }^{10}$, coexistencia de generaciones con valores y principios muy dispares, aumento de la vigilancia pública sobre los derechos de los pacientes, 
aumento de la oferta de programas de formación en cirugía, mayores presiones del modelo económico de la salud privada ${ }^{11}$, restricciones horarias en las residencias del Reino Unido y EE.UU. ${ }^{12}$, sociedad más educada, mejor informada y más atenta a las necesidades y derechos de su propia salud, etc. ${ }^{5}$. En suma, diversos fenómenos asociados a que la "oportunidad quirúrgica" en los programas de formación fuera cada vez más escasa y delicada desde el punto vista ético-moral.

Los médicos en general dejaron de ser aceptados como autoridad hasta el punto de cuestionarse su capacidad para supervisar la propia práctica y controlar la educación de sus profesionales. Se reconfiguró la relación entre los médicos, los pacientes y la sociedad. Tanto el desarrollo tecnológico, como los cambios sociopolíticos y la percepción pública de los médicos es lo que habitualmente se reconoce como los precipitantes del cambio del modelo educativo.

La demanda educacional en este nuevo contexto, se vio en la necesidad de migrar desde "lo que siempre se ha hecho" a "lo que mejor funciona para formar profesionales". En esta misma línea, el foco de la educación quirúrgica cambió desde entrenar una forma rígida para la realización de un conjunto de procedimientos establecidos a preparar al residente o estudiante, a tener la capacidad de adaptarse a un mundo sometido a cambios constantes y acelerados. Coincidentemente, este es precisamente el cambio de foco de la innovación en casi todas las áreas de educación superior en los tiempos actuales.

La puerta de entrada más importante de los cirujanos a la educación fue a través de la simulación quirúrgica en laparoscopía ${ }^{13}$. La gran mayoría de las publicaciones relacionadas con aspectos educativos de la cirugía son precisamente en esta área. Se logró demostrar que los programas de entrenamiento en distintos procedimientos simulaban lo que pretendían simular ${ }^{14,15}$, eran capaces de entrenar habilidad técnica ${ }^{16,17}$ y lo que es más importante, esto era transferible al pabellón quirúrgico con un impacto positivo en la seguridad de los pacientes ${ }^{18-20}$.

Sucesivamente, los cirujanos tuvimos que expandir nuestras habilidades profesionales a un repertorio diferente y más amplio en torno a conceptos pedagógicos y teoría educacional, lo cual es complejo de compatibilizar desde el abanico de competencias y responsabilidades que la especialidad demanda. De esto se desprende quizás el mayor desafío para la innovación en docencia quirúrgica.

Otra dificultad importante de considerar es el hermetismo con el que habitualmente hemos funcionado los cirujanos. Para realizar cambios significativos en educación quirúrgica, se ha hecho cada vez más evidente la necesidad del ingreso de otros profesionales a nuestro círculo para aprender de ellos y establecer alianzas colaborativas. No es extraño pensar entonces, que compartir parte del quehacer al cual uno estaba acostumbrado hacer y mediante el cual se formó, sea fuente de incomodidad y rechazo. No obstante, si nuestro objetivo es una educación quirúrgica innovadora, debemos estar dispuestos a este sacrificio y asumir el abismo que el progreso mismo ha producido entre ser cirujano y educador quirúrgico ${ }^{21}$.

\section{Análisis y recomendaciones}

$\mathrm{Si}$ bien en ciertos aspectos educacionales, los cirujanos chilenos hemos estado a la vanguardia gracias a la creación y contribución de laboratorios de habilidades quirúrgicas como el Centro de Simulación y Cirugía Experimental de la Pontificia Universidad Católica de Chile o el Centro de Entrenamiento en Habilidades Quirúrgica de la Universidad de Chile (CEHaQ), todavía queda mucho por hacer. Esto se basa en que la mayoría de lo que se ha hecho es implementar, desarrollar e investigar la validez, en su amplia definición, de modelos de simulación que permitan entrenar habilidad técnica en los distintos procedimientos más utilizados en la cirugía actual. Sin embargo, aun estando en la vanguardia en este aspecto en particular, no hemos podido lograr que estos módulos de entrenamiento sean incorporados en forma amplia a los currículos de los diferentes programas de formación de nuestro país. Sólo a modo de ejemplo, incluso en esta área en que podríamos considerarnos aventajados, existe un importante desfase con países líderes como EE.UU. donde la implementación del programa formación en habilidades laparoscópica (FLS: Fundamentals of Laparoscopic Surgery) ${ }^{22}$ es requisito obligatorio para el ejercicio de la especialidad hace ya más de 10 años ${ }^{23}$.

Otro de los aspectos que podría criticarse sobre la simulación en cirugía tanto en el mundo como en nuestro país, es que el desarrollo, innovación e investigación en esta área, ha tenido un fuerte énfasis en la simulación laparoscópica y se ha privilegiado ampliamente el postgrado por sobre el pregrado. De esta forma, se han dejado inexploradas otras áreas de la simulación, importantísimas para el desarrollo profesional, como el manejo de crisis y desarrollo de habilidades no técnicas ${ }^{24}$.

Hoy en día, las habilidades no técnicas tales como la comunicación, toma de decisiones, colabo- 
ración, liderazgo y profesionalismo, forman parte únicamente del currículo oculto de los programas de formación de especialistas en nuestro país. Sin embargo, un vasto y creciente volumen de evidencia científica ha demostrado que estas destrezas son esenciales en la práctica de la especialidad y su falencia importante predictor de complicaciones quirúrgicas $^{25-27}$.

El verdadero desafío, es entonces, que los cirujanos nos hagamos cargo de profesionalizar los aspectos educacionales de la cirugía en toda su amplitud. Es decir, debemos ser capaces de establecer los perfiles de egreso de los distintos programas de cirugía en forma coordinada en base a las necesidades de la sociedad y sus proyecciones, tomando en cuenta la fluidez de los tiempos modernos y las necesidades de adaptabilidad. Si bien deben regularse los programas de residencia y asignaturas de pregrado en torno a estos perfiles de egreso, es fundamental que los distintos centros formadores en particular mantengan su autonomía, sello y mística. Esto es quizás tan importante como la existencia de estándares nacionales.

Creo necesario también mencionar que deben formarse organizaciones o asociaciones que revisen y trabajen sobre estos conceptos y evalúen la factibilidad, responsabilidad y proyección académica de los cambios innovadores, para hacer recomendaciones generales. Estas mismas, deben ser quienes regulen las competencias básicas que deben tener todos los programas de formación quirúrgica en Chile, como lo hace en el extranjero la $\mathrm{RACS}^{28}, \mathrm{AGCME}^{29}$, $\mathrm{APDS}^{29}, \mathrm{GMC}^{30}$ o CanMeds ${ }^{31}$, que regulan la formación abarcando todos los aspectos de la formación quirúrgica y no sólo lo teórico y lo técnico.

Es muy importante también reconocer la necesidad de que ciertos cirujanos debamos profesionalizarnos en educación y aplicar los marcos conceptuales de la teoría educativa moderna en nuestros programas. Debe cambiarse el enfoque educacional de aquel centrado en el profesor a uno centrado en el estudiante y aplicarse las teorías de educación más influyentes de la actualidad ${ }^{32}$. Esto implica realizar el diseño instruccional incorporando conceptos fundamentales como el de práctica deliberada, retroalimentación, simulación, herramientas de evaluación estándares como DOPS u OSATS ${ }^{33}$, comunidades de práctica, práctica reflexiva, etc.

Además, debemos fomentar que los currículos de formación de nuestro país cumplan ciertos estándares mundiales mínimos de la educación quirúrgica basada en simulación, como incorporar de forma obligatoria los módulos básicos de laparoscopía o el currículo de habilidades quirúrgicas para residen- tes (Surgical skills curriculum for residents) de la Asociación de Directores de Programas de Cirugía $(\mathrm{APDS})^{34}$.

Debido a la clara evidencia disponible sobre el impacto de las habilidades no técnicas del cirujano en los resultados postoperatorios de sus pacientes, que se incorporen las habilidades no técnicas a los programas de formación no sólo es aconsejable, sino que es una obligación moral. Estas deben ser incluidas en los currículos explícitos de los programas de residencia para que puedan ser enseñadas, ejercitadas y evaluadas. Una muy buena manera de poner esto en práctica, es mediante la incorporación de actividades de simulación y reflexión, en escenarios clínicos de complicaciones.

El último esfuerzo por estandarizar los programas de residencia quirúrgica en nuestro país, es una publicación del Dr. Juan Hepp del año 2008 en esta misma revista ${ }^{35}$. Han pasado ya 12 años desde esa fecha. Sólo como referencia, desde ese entonces, se ha producido en el mundo más del $70 \%$ de la evidencia disponible sobre educación quirúrgica junto con publicarse los dos libros de referencia más importantes en esta materia ${ }^{36,37}$.

Si bien hay que reconocer que la Sociedad de Cirujanos de Chile ha hecho esfuerzos importantes por difundir su visión en cuanto a la formación del cirujano en nuestro país y trazar líneas de trabajo y definir los desafíos más importantes ${ }^{39,40}$, no se han implementado, a mi parecer, planes concretos y radicales para que la educación en cirugía en nuestro país se profesionalice, se establezcan asociaciones de centros formadores y se establezcan estándares mínimos en los programas de formación.

\section{Conclusiones}

La innovación en docencia quirúrgica en nuestro país tiene hoy en día una sola prioridad: Profesionalizar la educación quirúrgica tanto a nivel de pregrado como postgrado. Es perentorio que la Sociedad de Cirujanos de Chile sea líder en esta labor e influya en los cambios de visión que se requieren, ya que hoy en día para los cirujanos no resulta atractivo ni desde el reconocimiento profesional ni desde el punto de vista económico.

Los distintos centros formadores deben preocuparse de que algunos miembros de su equipo se entrenen como formadores y de que esto se vaya perpetuando en el tiempo. Se deben crear o proponer programas de formación de educadores quirúrgicos avalados por las sociedades científicas y fundar agrupaciones de centros formadores que articulen y regulen los cambios educacionales que se requie- 
ren. Si bien es evidente que el cambio significa un terremoto del paradigma educativo actual, sólo ahí vamos a poder empezar a hablar realmente de Docencia Innovadora en Cirugía.

En base a la evidencia disponible, cuando hayamos prestado atención a las necesidades de la sociedad, incorporado conceptos educacionales modernos a los currículos, revisado las experiencias de países líderes, seguido los ejemplos de asociaciones internacionales, formado asociaciones locales y entrenado a nuestros especialistas en educación médica, vamos a estar recién comprendiendo cuál es el perfil del médico general o cirujano que debemos formar. Una vez logrado esto, estaremos ya en condiciones de buscar las herramientas que se necesitan para lograrlo. Si como resultado, encontramos que es relevante la implementación de sistemas altamen- te tecnológicos como realidad virtual, escenarios complejos de simulación, impresión $3 \mathrm{D}$, simulación en cirugía robótica, educación online, aplicaciones móviles entre otras ${ }^{38}$, sólo entonces será prudente, enfocarse en la tecnología.

\section{Responsabilidades éticas}

Protección de personas y animales. Los autores declaran que para esta investigación no se han realizado experimentos en seres humanos ni en animales.

Confidencialidad de los datos. Los autores declaran que en este artículo no aparecen datos de pacientes.

Conflictos de interés: no hay.

\section{Bibliografía}

1. Brennan J, Ryan S, Ranga M, Broek S, Durazzi N, Kamphuis B. Study on innovation in higher education: final report. European Commission Directorate for Education and Training Study on Innovation in Higher Education. 2014. Publications Office of the European Union. Luxembourg.

2. Publication years by topic. Web of Science. Disponible en: http://wcs. webofknowledge.com/RA/analyze.do? product $=$ WOS \&SID $=5 \mathrm{~B} 8 \mathrm{nTf} 3 \mathrm{D} 8 \mathrm{bYrE}$ 5XDcvh\&field=PY_PublicationYear PublicationYear_en\&yearSort=true. Acceso el 28 de marzo de 2020.

3. Surgical Education. Imperial College, London. Disponible en: https://www. imperial.ac.uk/study/pg/medicine/surgicaleducation/. Acceso el 28 de marzo de 2020.

4. Master of Surgical Education. University of Melbourne. Disponible en: https:// study.unimelb.edu.au/find/courses/ graduate/master-of-surgical-education/. Acceso el 28 de marzo de 2020.

5. Kneebone, R. Surgical Education: A Historical Perspective. En Advancing Surgical Education. Springer Singapore 2019. https://doi.org/10.1007/978-981-133128-2 2.

6. Martínez, J. La evolución de la enseñanza quirúrgica: un desafío permanente. Rev Chil Cir. 2018;70:201-2.
7. Carter B. The fruition of Halsted's concept of surgical training. Surgery 1952;32:51827.

8. Kohn L, Donaldson M. To Err Is Human: Building a Safer Health System. Washington, DC: National Academy Press 2000.

9. Smith, R. All changed, changed utterly. British medicine will be transformed by the Bristol case. BMJ 1998;316:1917-8. https://doi.org/10.1136/ bmj.316.7149.1917.

10. Fry H. Educational Ideas and Surgical Education. En Advances in Medical Education (pp. 19-36). Springer Netherlands 2011. https://doi. org/10.1007/978-94-007-1682-7_2.

11. Reznick R, MacRae H. Teaching Surgical Skills: Changes in the Wind, N Engl J Med 2006;355:2664-9. https://doi. org/10.1056/NEJMra054785.

12. The Working Time Regulations. Health and Safety Executive, disponible en: https://www.hse.gov.uk/contact/faqs/ workingtimedirective.htm. Acceso el 28 de marzo de 2020

13. Zendejas B, Brydges R, Hamstra S, Cook D. State of the Evidence on Simulation-Based Training for Laparoscopic Surgery. Ann Surg. 2013;257:586-93. https://doi.org/10.1097/ sla.0b013e318288c40b.

14. Scott D, Bergen P, Rege R, Laycock R, Tesfay S, Valentine R, et al. Laparoscopic training on bench models: better and more cost effective than operating room experience? J Am Coll Surg. 2000;191:272-83. https://doi.org/10.1016/ S1072-7515(00)00339-2.

15. Rosser J, Rosser L, Savalgi R. Skill acquisition and assessment for laparoscopic surgery. Arch Surg. 1997;132:200-4. https://doi.org/10.1001/ archsurg.1997.01430260098021.

16. Derossis A, Fried G, Abrahamowicz M, Sigman H, Barkun J, Meakins J. Development of a model for training and evaluation of laparoscopic skills. Am J Surg. 1998;175:482-7. https://doi. org/10.1016/S0002-9610(98)00080-4.

17. Brunner W, Korndorffer J, Sierra R, Dunne J, Yau C, Slakey D, et al. Determining standards for laparoscopic competency using virtual reality. Am Surg. 2005;71:1-7.

18. Varas J, Mejía R, Riquelme A, Maluenda F, Buckel E, Salinas J, et al. Significant transfer of surgical skills obtained with an advanced laparoscopic training program to a laparoscopic jejunojejunostomy in a live porcine model: feasibility of learning advanced laparoscopy in a general surgery residency. Surg Endosc. 2012;26:3486-94. https://doi.org/10.1007/s00464-012-23914.

19. Sroka G, Feldman L, Vassiliou M, Kaneva P, Fayez R, Fried G. Fundamentals of Laparoscopic Surgery simulator training to proficiency improves laparoscopic performance in the operating room- a 
randomized controlled trial. Am J Surg. 2000;199:115-20. https://doi. org/10.1016/j.amjsurg.2009.07.035.

20. Anastakis D, Regehr G, Reznick R, Cusimano, Murnaghan J, Brown M, et al. Assessment of technical skills transfer from the bench training model to the human model. Am J Surg. 1999;177:16770. https://doi.org/10.1016/s00029610(98)00327-4.

21. Bleakley A. Learning and Identity Construction in the Professional World of the Surgeon. En: Fry H, Kneebone R. (eds) Surgical Education. Advances in Medical Education 2001, vol 2. Springer, Dordrecht. https://doi.org/10.1007/97894-007-1682-7 11.

22. Peters J, Fried $\bar{G}$, Swanstrom L, Soper N, Sillin L, Schirmer B, et al. Development and validation of a comprehensive program of education and assessment of the basic fundamentals of laparoscopic surgery. Surgery 2004;135:21-7. https://doi.org/10.1016/ s0039-6060(03)00156-9.

23. The American Board of Surgery. Training Requirements. Disponible en: http:// www.absurgery.org/default.jsp?certgsqe training. Acceso el 28 de marzo de 2020.

24. Anthony A, Muralidharan V. The Contemporary Context of Surgical Education. En: Nestel D, Dalrymple K, Paige J, Aggarwal R. (eds) Advancing Surgical Education. Innovation and Change in Professional Education 2019, vol 17. Springer, Singapore. https://doi. org/10.1007/978-981-13-3128-2_3.

25. Hull L, Arora S, Aggarwal R, Darzi A, Vincent C, Sevdalis N. The impact of non-technical skills on technical performance in surgery: A systematic review data sources. J Am Coll
Surg. 2012;214:214-30. https://doi. org/10.1016/j.jamcollsurg.2011.10.016.

26. Cooper W, Guilillamondegui O, Hines O, Hultman C, Kelz R, Shen P, et al. Use of unsolicited patient observations to identify surgeons with increased risk for postoperative complications. JAMA Surg. 2017;152:522-29. https://doi.org/10.1001/ jamasurg.2016.5703.

27. Bearman M, O’Brien R, Anthony A, Civil I, Flanagan B, Jolly B, et al. Learning surgical communication, leadership and teamwork through simulation. J Surg Educ. 2012;69:201-7. https://doi. org/10.1016/j.jsurg.2011.07.014.

28. RACS. Diversity \& inclusion plan. Royal Australasian College of Surgeons 2016. Disponible online en: https://ama.com. $\mathrm{au} /$ sites/default/files/documents/RACS diversity_and_inclusion_plan.pdf. Acceso el 28 de marzo de 2020.

29. Leach DC. A model for GME: shifting from process to outcomes. A progress report from the Accreditation Council for Graduate Medical Education. Med Educ 2004;38:12- 4. https://doi.org/10.1111/ j.1365-2923.2004.01732.x.

30. GMC. Promoting excellence: Standards for medical education and training. General Medical Council 2015. Disponible online en: gmc-uk.org/-/ media/documents/Promoting_excellence standards_for_medical_education_and training_0715.pdf_61939165.pdf. Acceso el 28 de marzo de 2020.

31. Royal College of Physicians and Surgeons of Canada. About CanMEDS, disponible online en: http://www.royalcollege.ca/ rcsite/canmeds/about-canmeds-e, acceso el 28 de marzo de 2020.

32. Chauvin S. Applying Educational Theory to Simulation-Based Training and Assessment in Surgery. Surg Clin North Am. 2015;95:695-715. https://doi. org/10.1016/j.suc.2015.04.006.

33. Martin J, Regehr G, Reznick R, MacRae $\mathrm{H}$, Murnaghan J, Hutchison C, et al. Objective structured assessment of technical skill (OSATS) for surgical residents. Br J Surg. 1997:84:2738. https://doi.org/10.1046/j.13652168.1997.02502.x.

34. Scott D, Dunnington G. The new ACS/ APDS skills curriculum: moving the learning curve out of the operating room. J Gastrointest Surg. 2008;12:213-21. https://doi.org/10.1007/s11605-0070357-y.

35. Hepp J, Csendes A, Ibáñez F, Llanos O, San Martín S. Programa de la especialidad Cirugía General. Definiciones y Propuestas de la Sociedad de Cirujanos de Chile. Rev Chil Cir. 2008;60:79-85.

36. Fry H, Kneebone R. (Eds). Surgical Education: Theorizing an Emerging Domain. Dordrecht New York: Springer 2011.

37. Nestel D, Dalrymple K, Paige J, Aggarwal R (Eds). Advancing Surgical Education: Theory, Evidence and Practice, Singapore: Springer, 2019.

38. Badash I, Burtt K, Solorzano C, Carey J. Innovations in surgery simulation: a review of past, current and future techniques. Ann Transl Med. 2016;4:463-7. https://dx.doi. org/10.21037\%2Fatm.2016.12.24.

39. Bustamante M. Desafíos en la formación del cirujano. Rev Chil Cir. 2015;67:348-9.

40. Bustamante M, Espinoza R, Hepp J, Martínez J. Estándares de la Formación del Cirujano. Visión de la Sociedad de Cirujanos de Chile. Rev Chile Cir. 2015;67:102-8. 